\title{
COMMENTARY
}

\section{Dysnatremia and mortality: do sweat the small stuff...}

\author{
John R Klinck', Lisa McNeill/2,3 and David K Menon",2,* \\ See related research by Darmon et al., http://ccforum.com/content/17/1/R12
}

\begin{abstract}
Marked dysnatremia is associated with increased mortality in patients admitted to intensive care. However, new evidence suggests that even mild deviations from normal and simple variability of sodium values may also be significant. Should these findings prompt clinicians to re-evaluate the approach to fluid management in this setting? Sodium disorders, on one hand, are known to result from overzealous administration or restriction of free water or sodium ions. However, they are also associated with a range of co-morbidities and drug treatments that alter water loss and sodium handling in the nephron independently of prescribed fluid regimens. Moreover, powerful neuroendocrine and inflammatory responses to surgery, trauma and other acute illness may induce or intensify such changes, altering the response to administered fluids. These observations suggest that both patient and treatment variables contribute, but the extent to which sodium disturbances are preventable and whether prevention improves outcome are unknown. Dysnatremia certainly reflects underlying systemic disorders, but how important is fluid management as a cause, and does it contribute independently to poorer outcomes through osmotic or other mechanisms? Although total fluid volume and doses of potassium and glucose are regularly adjusted in critically ill patients, sodium is usually delivered at standard concentrations as long as serum values lie within an acceptable range. It may be prudent to pay closer attention to these values, especially when abnormal, when fluctuating or when an adverse trend is present. More frequent measurements of sodium in blood, urine and drainage fluids, and appropriate adjustment of the sodium content of prescribed fluids, may be indicated. Until more light can be shed on the pathophysiology of dysnatremia in the critically ill, we should assume that better control of plasma sodium levels may yield better outcomes.
\end{abstract}

Moderate-to-severe dysnatremia has long been recognized as a marker of acuity and mortality risk in the critically ill patient. However, accumulating evidence, including the work of Darmon and colleagues in the previous issue of Critical Care [1], suggests that even mild abnormalities of serum sodium concentration present on ICU admission predict increased 30-day mortality. Other recent work indicates that even variability of sodium concentrations, including changes within the normal range, is linked to an increased risk of death [2]. How should the clinician respond to these findings? Does normalization or stabilization of serum sodium values improve outcomes? Will control of serum sodium be the

*Correspondence: dkm13@wbic.cam.ac.uk

2Division of Anaesthesia, University of Cambridge, Box 93, Addenbrooke's Hospital, Cambridge CB2 2QQ, UK

Full list of author information is available at the end of the article next important quality measure in the critical care setting [3]?

First, a note of caution. Large observational studies, made possible by growth of critical care services, multicenter collaborations and electronic capture of huge quantities of data, are ever more able to identify predictive effects from subtle changes in laboratory and physiological parameters. In the case of serum electrolytes, however, unmeasured confounders such as blood glucose and disparate or imprecise laboratory assays may become important when small changes are evaluated. Frequency of measurement, naturally higher in sicker patients, is another potential source of bias. It is important not to over-interpret these findings in assessing prognosis and in pursuing interventions. Note that the predictive impact of sodium values near the limits of the normal range in the study from Darmon and colleagues [1] did not achieve statistical significance. On the other hand, the adverse prognostic significance of even modest deviations from the normal range is clear 
[4]. What cannot be determined from existing studies is the extent to which these dysnatremias are preventable, and whether prevention or correction improves outcome.

That sodium disorders caused by iatrogenic free water excess and loss are preventable is self-evident. However, it is also clear that these disorders are associated with a range of co-morbidities and drug treatments that alter water loss and sodium retention in the nephron independently of fluid replacement, and that processes distinct from the consequent electrolyte abnormalities may be responsible for biological effects. In patients presenting to the ICU, dysnatremias are often associated with powerful hormonal responses to injury and inflammation, with effects on osmotic and non-osmotic regulation of antidiuretic hormone [5] (causing a syndrome of inappropriate antidiuresis), the renin-angiotensin-aldosterone axis [6], and the release of natriuretic peptides [7]. Yet little is known about how these responses are altered by administration of fluids, or how changes in sodium concentration might be anticipated from clinically measurable parameters.

Although doses of potassium, glucose and even water are regularly adjusted, sodium is usually delivered at standard concentrations as long as serum values lie within an acceptable range. This is routine practice in renal replacement therapies, and in many ICU patients, with little consideration of the last-recorded sodium measurement or of easily measured sodium losses in urine and drains. While this is well tolerated in most individuals, in some the sodium content of prescribed fluids may be poorly matched to their intravascular volume and neuroendocrine condition, leading to a failure of sodium homeostasis. Until dysnatremia is shown to be a non-causal biomarker of a harmful underlying systemic process [6], it may be prudent to assume that even modest sodium fluctuations and attendant osmotic shifts are inherently undesirable, and that refined control of plasma sodium levels offers scope for better outcomes.

To minimize this iatrogenic element, to define appropriate therapies and to confirm their beneficial effects, several key investigative and therapeutic steps are needed. Measurements of both serum sodium and of sodium ions lost from the body need to be performed more frequently, especially when an abnormal serum concentration or adverse trend is present. Detailed data on risk-associated conditions and linked fluid and pharmacologic interventions should be collected and analyzed to inform the development of more sophisticated, individualized fluid regimes. Finally, these more tailored regimes should be tested in randomized trials. Only such studies can conclusively show whether mild dysnatremia is a remediable contributor to mortality risk, or a marker of impaired neuroendocrine homeostasis, insensitive to treatment and predisposing to allied causes of organ failure and death.

Competing interests

The authors declare that they have no competing interests.

\section{Author details}

'Directorate of Perioperative Care, Box 93, Addenbrooke's Hospital, Cambridge CB2 2QQ, UK. ²Division of Anaesthesia, University of Cambridge, Box 93, Addenbrooke's Hospital, Cambridge CB2 2QQ, UK. 'Department of Public Health, University of Cambridge, Strangeways Research Laboratory, Worts Causeway, Cambridge, CB1 8RN, UK.

Published: 2 April 2013

\section{References}

1. Darmon M, Diconne E, Souweine B, Ruckly S, Adrie C, Azoulay E, Clec'h C, Garrouste-Orgeas M, Schwebel C, Goldgran-Toledano D, Khallel H, Dumenil AS, Jamali S, Cheval C, Allaouchiche B, Zeni F, Timsit JF: Prognostic consequences of borderline dysnatremia: pay attention to minimal sodium change. Crit Care 2013, 17:R12.

2. Sakr Y, Rother S, Ferreira AM, Ewald C, Dunisch P, Riedemmann N, Reinhart K: Fluctuations in serum sodium level are associated with an increased risk of death in surgical ICU patients. Crit Care Med 2013, 41:133-142.

3. Peruzzi W: Sodium balance: the next core measure? Crit Care Med 2013, 41:351-352.

4. Funk GC, Lindner G, Druml W, Metnitz B, Schwarz C, Bauer P, Metnitz PG: Incidence and prognosis of dysnatremias present on ICU admission. Intensive Care Med 2010, 36:304-311.

5. Russell JA: Bench-to-bedside review: Vasopressin in the management of septic shock. Crit Care 2011, 15:226.

6. Doerschug KC, Delsing AS, Schmidt GA, Ashare A: Renin-angiotensin system activation correlates with microvascular dysfunction in a prospective cohort study of clinical sepsis. Crit Care 2010, 14:R24.

7. Wang F, Wu Y, Tang L, Zhu W, Chen F, Xu T, Bo L, Li J, Deng X: Brain natriuretic peptide for prediction of mortality in patients with sepsis: a systematic review and meta-analysis. Crit Care 2012, 16:R74

doi:10.1186/cc12560

Cite this article as: Klinck JR, et al.: Dysnatremia and mortality: do sweat the small stuff... Critical Care 2013, 17:128. 